\title{
Relation aux sujets en recherche biographique en éducation
}

\section{RÉSUMÉ}

Cette contribution se propose d'interroger le rapport du chercheur aux acteurs-sujets de la recherche, au regard des spécificités de la recherche biographique en éducation et les effets des dispositifs qu'elle met en œuvre. Elle prendra appui pour ce faire sur une recherche menée auprès de plusieurs groupes de collégiens à partir d'entretiens biographiques collectifs et individuels et de récits écrits concernant leur parcours scolaire. Cette recherche avait deux finalités: comprendre les modalités de subjectivation de leur parcours scolaire et d'orientation ; transformer une situation dans laquelle les élèves avaient des difficultés à s'approprier leur parcours d'orientation et à coopérer. Il s'agira de présenter le dispositif mis en œuvre et son cadre épistémologique et méthodologique puis d’en analyser les effets pour comprendre les enjeux de la relation du chercheur aux acteurs-sujets en recherche biographique. Il sera mis en évidence que les spécificités de ce champ de recherche invitent, d'une part, à inscrire l'investigation dans un espace de rencontre symétrique, espace d'auteurisation, propice au développement du pouvoir d'agir des acteurs, et, d'autre part, à développer une posture empathique garante du respect des acteurs et de leur autonomisation.

Mots-clés: Recherche biographique en éducation; Posture; Sujet; Pouvoir d'agir.

\section{INTRODUCTION}

La recherche biographique en éducation (RBE) confronte le chercheur à des questionnements éthiques spécifiques dans la mesure où, en plus d'étudier " les modes de constitution de l'individu en tant qu'être social singulier » (Delory-Momberger, 2014, p. 74), « elle invite les hommes à réhabiliter ce qui construit l'humanité de l'individu : l'expérience, l'historicité et la quête de sens » (M'Biatong, 2019, p. 144).

En effet, parce qu'elle s'inscrit dans une perspective de transformation et d'émancipation qui altère ${ }^{1}$ les participants comme le chercheur, elle questionne les interactions entre la recherche et le processus de formation du chercheur. Dans ce cadre général, ma contribution viendra interroger plus
Anne Dizerbo ${ }^{i}$

Université Sorbonne Paris Nord, France
1. «Altérer » est ici envisagée comme « le processus, à partir duquel un sujet change (devient autre) sans, pour autant, perte de son identité, en fonction d'influences (qui peuvent évidemment être perçues comme, tout à la fois, négatives et positives) exercées par un autre (ou par d'autres) » (Ardoino, 2000, L'altération, p. 195-198) 
particulièrement la relation du chercheur aux personnes qu'il rencontre lorsqu'il met en œuvre une recherche inscrite dans la RBE, en fonction de la spécificité des savoirs produits dans ce champ.

Pour ce faire, je m'appuierai sur la recherche menée dans le cadre de mon doctorat en sciences de l'éducation, entre 2009 et 2012. Elle portait sur les modalités de subjectivation du parcours scolaire et d'orientation d'élèves inscrits en classe de $4^{\text {ème}}$, âgés de 13 à 15 ans, et elle visait à la fois à produire des connaissances sur ces modalités et à transformer une situation insatisfaisante pour les élèves comme pour l'enseignante et chercheure.

Dans un premier temps, je présenterai cette recherche (la situation qui l'a générée, ses acteurs, ses visées). J'exposerai ensuite mes choix épistémologiques et méthodologiques. Enfin, après avoir proposé non pas les résultats complets de la recherche mais plutôt ceux qui concernent ses effets sur les acteurs, je les mettrai en discussion au regard de la relation établie avec les élèves dans le cadre de cette recherche et de ses finalités, interrogeant ma posture de chercheure.

\section{PRÉSENTATION DE LA RECHERCHE}

J'ai entamé cette recherche en 2009, alors que j'étais professeure de français, dans un collège classé en ZEP (Zone d'Éducation Prioritaire). Je venais de reprendre des études en sciences de l'éducation, cherchant à mieux comprendre le système éducatif et à améliorer ma pratique.

Je m'interrogeais notamment sur mon enseignement dans une classe expérimentale dont j'étais responsable, en tant que professeure principale. J'y accompagnais des élèves de $4{ }^{\text {ème }}$, niveau problématique car il invitait encore à ce moment-là à une première réflexion sur l'orientation et pouvait conduire à quitter la filière générale en vue d'une professionnalisation.

Il s'agissait dans cette classe constituée d'élèves en situation de difficulté, souvent en conflit avec l'institution scolaire et ses acteurs, de prévenir le décrochage, en instaurant un meilleur climat de classe, et en luttant contre le désintérêt manifeste des élèves pour leur orientation. Ils le justifiaient le plus souvent par le sentiment de ne pas vraiment pouvoir choisir cette orientation, la décision appartenant aux adultes (équipe éducative et parents).

À court terme, je craignais leur déscolarisation avant l'obtention des qualifications et certifications leur permettant de s'inscrire sur le marché de l'emploi, phénomène fréquemment constaté au cours de mon expérience d'enseignante. À plus long terme, le risque me semblait important que le rapport conflictuel dans lequel ils avaient inscrits leur scolarité ne trouve des prolongements dans un rapport conflictuel durable aux situations de formation, et que la mauvaise estime d'eux-mêmes développée à l'école ne les mènent à des comportements d'« autocensure ». Dominicé (1999), notamment, avec son équipe à l'Université de Genève, a démontré que le rapport à la formation et la compétence d'apprendre au cours d'une vie sont liés à l'expérience première de la scolarité.

Je développais le sentiment de ne pas assurer convenablement mes missions et je m'interrogeais sur le sens de mon métier et, plus largement, de 
l'éducation. Je développais, à l'instar de mes élèves, un sentiment de ne pas pouvoir agir qui fragilisait mon identité d'enseignante.

Tap (1980) envisage l'identité comme « l'ensemble des représentations et des sentiments qu'une personne développe à propos d'elle-même, comme ce qui permet de rester le même, de se réaliser soi-même et de devenir soi-même, dans une société et une culture donnée, et en relation avec les autres » (p. 57). La situation que nous partagions mes élèves et moi mettait manifestement à l'épreuve notre sentiment d'identité.

Je refusais de me résoudre à une forme de fatalité allant à l'encontre des principes égalitaires que je défendais en entrant dans l'Éducation nationale. Reprendre des études et entrer en recherche répondait donc au double objectif de mieux comprendre les enjeux identitaires liés à nos expériences respectives d'élèves et d'enseignante et de transformer la situation pour nous permettre de sortir du sentiment d'impuissance à agir qui était le nôtre, qu'il concerne la subjectivation du parcours scolaire et d'orientation de mes élèves ou la subjectivation de mon propre parcours professionnel.

Cela impliquait, pour mieux la prendre en compte, de rendre visible l'expérience des élèves. C'est donc tout naturellement que j'ai inscrit ma recherche dans le champ de la RBE.

\section{CADRE ÉPISTÉMOLOGIQUE ET MÉTHODOLOGIQUE}

\subsection{LA RECHERCHE BIOGRAPHIQUE EN ÉDUCATION}

Le paradigme du biographique, au fondement de la RBE, s'inscrivant dans la perspective de la Bildung allemande, vise l'émergence d'un sujet réflexif, lucide et responsable de ses choix et en ce sens " auteur » de son parcours. J'adopte ici le sens d'« auteur » proposé par Ardoino qui considère l'agent comme le «rouage dans la mécanique », l'acteur comme un "porteur de sens » qui choisit la manière d'interpréter son rôle et l'auteur comme le " créateur de son rôle » dont il produit le sens (Ardoino, 2000, p. 205-215).

Dans le paradigme de la RBE, le rôle ne se trouve pas dissocié de la personne qui le tient. Cela m'invitait donc à inscrire nos parcours d'élèves et d'enseignante dans nos parcours globaux et à sortir du rapport hiérarchique qu'introduit la situation d'enseignement pour constituer dans la situation de recherche un espace horizontal de rencontre et de construction partagée de connaissances. Pour accompagner les élèves dans le développement de leur pouvoir d'agir et transformer la situation problématique dans laquelle nous étions impliqués, il était nécessaire de ne pas construire un dispositif de recherche dans lequel ils se trouveraient en position d'informateurs. Il s'agissait plutôt de leur proposer une position de sujets-auteurs, en commençant par leur proposer d'être auteur d'un récit de leur parcours.

Ricœur (1990) considère que c'est par la médiation de la narration que se construit le sentiment d'identité de la personne. Pour lui, la vie n'a de forme, elle n'atteint à l'unité d'une « totalité singulière » que lorsqu'elle est racontée (Ricœur, 1990, p. 190). C’est aussi ce qu'affirme Larrosa: « Cela signifie que le moi, qui est dispersion et activité, se constitue en tant qu'unité de sens 
pour lui-même dans la temporalité d'une histoire, d'un récit » (Larrosa, 1998, p. 95). Il précise encore: « L'autocompréhension narrative se produit dans ce gigantesque bouillonnement d'histoires qu'est la culture et avec laquelle nous organisons notre propre expérience (le sens de ce qui nous arrive) et notre propre identité (le sens de ce que nous sommes) » (ibid).

C'est donc en s'appuyant sur des modèles narratifs que l'homme donne forme à ses expériences et construit une interprétation du vécu qui constitue l'un des objets de la RBE : « les modèles narratifs servent de patrons biographiques aux constructions individuelles. Ils procurent aux individus des schémas et des modalités de relation à eux-mêmes et d'insertion au sein de la collectivité, et ils accompagnent les évolutions sociétales dans ce domaine » (Delory-Momberger, 2009, p. 33).

Mais au-delà de ces fonctions formatives et interprétatives, le récit possède également une fonction générative:

La construction de la personnalité ne semble pouvoir se faire sans cette capacité de se raconter. Mais lorsque nous sommes dotés de cette capacité, alors nous pouvons construire une personnalité qui nous relie aux autres, qui nous permet de revenir sur notre passé, tout en nous préparant à affronter un futur que nous imaginons. (Bruner, 2010, pp. 77-78)

L'imagination narrative permet de produire plusieurs fictions pour interpréter le passé mais aussi pour envisager l'avenir, versions multiples et ouvertes dont l'individu teste les potentialités avant d'en choisir une qui réponde aux besoins de la situation, en prenant en compte les interactions sociales qu'elles engagent (notre récit se fait aussi en fonction de ce que nous pensons de ce que penseront les autres). Grâce à la performativité du récit, le narrateur inscrit son existence dans un environnement social et culturel, dans la continuité de son histoire, dans une relation aux autres.

La médiation narrative, en s'inscrivant dans un processus d'autant plus puissant s'il concerne un groupe d'individus, rend possible la compréhension et l'interprétation de ses propres expériences et de celles des autres, et multiplie les possibilités d'interprétation des expériences à venir. L'objet construit d'un récit permet de se projeter et, par l'adresse ou le partage que l'on en fait, d'accéder à des formes renouvelées de socialisation et de reconnaissance qui autorisent la transformation des agir.

\subsection{LE DISPOSITIF ET LES ACTEURS}

C'est pourquoi j'ai choisi dans un premier temps de proposer aux élèves une narration socialisée de leur parcours scolaire.

Il est important de mentionner que le dispositif initial n'a pas été construit préalablement à sa mise en œuvre mais au cours de celle-ci. Par ailleurs, même si les grandes lignes en ont ensuite été reproduites avec les différents groupes d'élèves, il a fait l'objet de plusieurs adaptations, en fonction de leurs spécificités et de la situation. 
La recherche s'est déroulée sur trois années et s'est appuyée sur des entretiens menées avec des élèves inscrits en $4^{\text {ème }}$ dans cinq établissements différents (Tableau 1).

Tableau 1

Groupes d Élèves Concernés

\begin{tabular}{|c|c|c|c|}
\hline Année 1 & Annce 2 & Annce 2 & Anné 3 \\
\hline $\begin{array}{l}\text { Une classe experimeniale } \\
\text { de } 16 \text { élEves en situation } \\
\text { de dificulié dont j'érais } \\
\text { professeure de français et } \\
\text { professeur principal. }\end{array}$ & $\begin{array}{l}\text { Une classe de } 16 \\
\text { ćk'ves inscrite dans } \\
\text { un dispositif de } \\
\text { double-alicrnance } \\
\text { d'un stablissement } \\
\text { voisin dans lequel je } \\
\text { n'enseignais pos. }\end{array}$ & $\begin{array}{l}\text { Une elasse héérogìne de } \\
28 \text { elèves de tème à quil } \\
\text { j"enscignais le frunçais. }\end{array}$ & 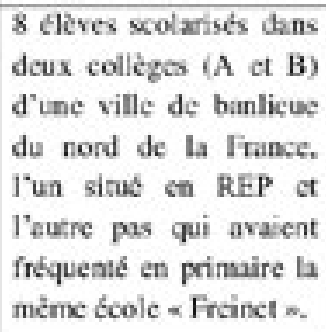 \\
\hline
\end{tabular}

\subsection{LE RECUEIL DES DONNÉES}

La première année, avec ma classe expérimentale, j'ai d'abord réuni les élèves et leur ai proposé de participer à ma recherche mais, plus encore, de s'y associer. Quinze d'entre eux ont accepté. La 16ème élève a finalement fait le choix de nous rejoindre à partir de la seconde étape. Je les ai invités à prendre la parole chacun à leur tour pendant une matinée banalisée pour raconter leur parcours scolaire et la manière dont ils en envisageaient la suite.

Dans un second temps, j'ai les invités à écouter l'enregistrement sonore de leur récit et à en produire une version écrite. J'attendais de ce passage par l'écrit, qui visait à constituer un recueil à destination de l'ensemble des élèves de la classe, qu'il ancre les élèves dans un statut d'auteur.

Enfin, j’ai proposé aux élèves qui le souhaitaient des entretiens individuels. Il s'agissait de solliciter encore une fois le récit de leur parcours scolaire, mais cette fois sans le soumettre aux regards des pairs et dans un moment plus long qui permettrait plus facilement une mise à distance et/ou un enrichissement interprétatif de l'expérience scolaire. Il s'agissait d'œuvrer à la construction d'une historicité (une articulation des trois dimensions temporelles de l'existence : passé, présent et avenir), qui permet d'entrer sans une démarche de construction l'élaboration d'un projet de vie.

Le cadre de la production de données a été co-élaboré avec les élèves. Les règles qui lui donnaient forme définissaient leurs attentes et droits en termes de :

- confidentialité (anonymisation des récits) ;

- propriété des données (non transmission des bandes sonores à d'autres que l'élève concerné) ;

- respect et d'écoute (il n'était pas possible de commenter l'expérience d'un autre élève mais il était possible d'exprimer un écho produit dans sa propre expérience) ;

- droit d'accès aux interprétations et aux résultats (toutes les données brutes, les interprétations et les résultats pouvaient être transmises aux élèves à leur demande afin qu'ils puissent les rectifier, les enrichir ou les commenter). 
La seconde année, avec la classe hétérogène de 28 élèves du collège de ZEP à qui j'enseignais le français, voulant vérifier que les résultats obtenus n'étaient pas contextuels, j'ai rencontré une difficulté. J'ai voulu reconduire le dispositif à l'identique, mais, d'une part, je ne les ai pas invités à s'associer à la recherche et, d'autre part, j'ai omis l'étape de construction du cadre avec les élèves. Il en a découlé que les élèves ont été peu nombreux à prendre la parole, ont globalement contesté le dispositif et en ont interrogé le sens. Je me suis remise en cause devant cet échec, comprenant que je m'étais montrée peu respectueuse de leur personne. Convaincue des effets positifs du dispositif, j'avais occulté qu'ils étaient intimement liés à l'élaboration d'un espace symétrique de co-construction de connaissances dans lequel nous partagions les mêmes objectifs. Je me suis excusée auprès de cette classe et j'ai expliqué aux élèves ma démarche, leur proposant cette fois une association pour produire ensemble des connaissances. Ils se sont dès lors impliqués de la même manière que la classe précédente et le dispositif a pu être reconduit. Je l'ai complété par plusieurs moments de discussions concernant le vécu de la scolarisation et des contraintes qu'elles imposent, le rapport aux enseignants, l'orientation.

La troisième année, avec les deux petits groupes de collégiens ayant fréquenté en primaire la même école Freinet, j'ai reproduit les deux premières étapes du dispositif, en leur proposant de nouveau une position de co-chercheurs. Nous avons mis en œuvre le moment de narration socialisée et d'écriture. Mais il m'a été impossible de leur proposer des entretiens individuels, coûteux en temps et en organisation, en raison de l'éloignement géographique entre ce terrain de recherche et mon lieu d'enseignement. Il limitait les possibilités de rencontres avec les élèves. Par ailleurs, mes objectifs n'étaient plus les mêmes à la lueur des résultats des deux premières années de recherche. En effet, mon objectif n'était plus seulement de produire des connaissances sur les modalités de subjectivation du parcours scolaire et d'orientation, mais aussi de comprendre quels étaient les liens entre compétences narratives et compétences à s'orienter qui étaient apparus avec l'analyse des premières données. Les élèves manifestant des difficultés à construire un récit se trouvaient aussi en difficulté dans l'élaboration d'un projet d'orientation. C'est donc pour comprendre ce phénomène que j'avais souhaité rencontrer des élèves auxquels avait été dispensé un autre type d'éducation narrative que celui en vigueur dans l'enseignement traditionnel, basé essentiellement sur la fiction littéraire. Je m'étais tournée vers des élèves ayant bénéficié d'une pédagogie davantage axée sur l'expression de l'expérience personnelle. J'avais fait l'hypothèse que ce type d'éducation narrative pouvait permettre de développer des compétences narratives sur lesquelles prendre appui pour se mettre en projet. J'ai pu constater que ce n'était pas forcément le cas. Il apparaissait que les élèves scolarisés au collège en REP (Réseau d'Éducation Prioritaire) présentaient des difficultés à se mettre en récit et à se projeter, alors que ceux qui étaient scolarisés dans un établissement sans spécificité se montraient plus à l'aise. L'éducation narrative reçue en primaire n'avait donc pas d'effet sur leurs compétences.

Je voulais comprendre ce qui avait construit les différences entre les deux groupes d'élèves et je les ai confrontés à ma question dont ils se sont saisis. Ils m'ont fait la proposition de les réunir pour un entretien visant à y 
répondre collectivement. Je les ai donc invités à co-analyser avec moi les premières données constituées de la transcription de la narration de leurs écrits. Il s'agissait plus précisément de comprendre pourquoi, alors qu'ils avaient tous été scolarisés dans la même école (une école dite « Freinet»), ils construisaient des figures d'élèves différentes. Elles s'inscrivaient pour les élèves d'un des groupes, qui parvenaient à se projeter dans l'avenir, dans une conformité aux attentes de l'institution scolaire, et pour les élèves du second groupe, qui peinaient à élaborer un projet d'orientation, dans des relations conflictuelles avec cette institution.

\subsection{PLACE ET RÔLE DES ACTEURS DE LA RECHERCHE}

Pour mener à bien la recherche, l'incident qui s'est produit avec le second groupe en témoigne, il a été important que les élèves s'impliquent dans la recherche plutôt que d'y être impliqués. Ardoino (1992) opère une distinction intéressante entre "s'impliquer » et " être impliqué ». Il rappelle que, dans une acceptation d'ordre juridique, le caractère d'implication est établi par un tiers et exclut une référence à une volonté consciente. S'y oppose alors la notion d' « engagement », qui répond à une volonté et qui apparaît dans le fait de s'impliquer. Si tous les acteurs participant d'une situation s'y trouvent impliqués, qu'ils en aient conscience ou non, qu'ils s'y engagent ou non, le fait de s'impliquer témoigne d'un engagement volontaire. Mais pour autant, la situation de recherche induit des rôles différents, associés à des postures et des tâches différentes.

En ce qui me concerne, mon rôle et ma posture (cf. tableau 2) n'ont pas été les mêmes dans les entretiens collectifs et les entretiens individuels. Ils ont demandé à être conscientisés et théorisés, pour répondre à l'exigence éthique liée à mon engagement auprès de mes élèves qui m'obligeait à sortir de la relation pédagogique ordinaire dans le cadre du dispositif. En effet, en menant avec mes élèves des entretiens biographiques dans le cadre de ma recherche, je sortais du cadre strict de mes obligations professionnelles et je quittais mes postures éducatives contractuelles ou d'autorité telles qu'elles sont définies par Le Bouëdec (in Boutinet, 2007, pp. 175-178), pour adopter dans les moments d'entretiens collectifs une posture d'animatrice liée à ma posture de chercheure : je me suis mise au service des élèves qui produisent leur récit, me mettant à l'écoute de leur besoin d'être entendus et reconnus, sans participer directement mais en prenant en charge l'organisation et la régulation du groupe, tout en ne perdant pas de vue l'objectif qui était aussi de constituer une communauté de travail.

Mais lors des entretiens individuels, permettant davantage de travailler avec les élèves à la construction de leur historicité, c'est une posture d'accompagnatrice que j'ai adoptée. Boutinet (2007, p. 5-16) définit la figure contemporaine de l'accompagnateur comme individu qui se met au service d'un autre pour l'accompagner dans des transformations. C'est bien l'objectif que j'ai poursuivi en accueillant et en écoutant les récits de mes élèves tout en les aidant à discerner les points leviers de l'histoire scolaire qu'ils produisaient au cours des entretiens, les invitant par des relances à en développer le contexte. Ils étaient liés à moments de rupture dans la progression scolaire, 
associés le plus souvent à des épreuves biographiques (déménagements, divorces, décès), des relations difficiles avec certains enseignants, l'influence négative de certains camarades, ou des périodes de réussite associées à des rencontres avec certains enseignants, adultes ou camarades.

Dans les deux situations d'entretien, il ne s'agissait pas d'obtenir des réponses à des questions que je me posais en fonction des connaissances d'un contexte dont je disposais déjà et qu'elles viendraient illustrer. Les élèves auraient alors occupé une position d'enquêtés et produit un récit préfabriqué par mes questions. Mon questionnement ne précédait donc pas la narration des élèves mais lui succédait. J'avais la charge de les suivre alors qu'ils devenaient eux-mêmes producteurs de la question. Je recueillais les preuves de leur questionnement et m'attachais à percevoir les thèmes et les agencements présents dans leur narration pour comprendre les modalités singulières de subjectivation de leur parcours qui s'y déployaient.

Les élèves pour leur part avaient la charge d'effectuer un travail de mise en sens et en forme de leurs expériences qui les plaçaient en position « d'enquêter » sur eux-mêmes (Tableau 2).

Tableau 2

Rôle des Acteurs de la Recherche

\begin{tabular}{|c|c|c|}
\hline & Rôle des Alèves & Rôle du chercheur \\
\hline \multirow[t]{2}{*}{ Elapes 1 et 2 des années 1 et 2} & \multirow[t]{2}{*}{$\begin{array}{l}\text { Explorer leurs expériences et les } \\
\text { mettre en sens }\end{array}$} & $\begin{array}{l}\text { Organiser des temps de recueil des } \\
\text { donnces et d'interprétation } \\
\text { Animer le groupe, garantir le cadre } \\
\text { d'expression du vócu }\end{array}$ \\
\hline & & $\begin{array}{l}\text { Produire et proposer des } \\
\text { interpaétations }\end{array}$ \\
\hline Etape 3 des annees 1 et 2 & $\begin{array}{l}\text { Explorer leurs expériences et les } \\
\text { mottre en sens }\end{array}$ & $\begin{array}{l}\text { Accompagner les deves dans la } \\
\text { construction d'une historicité }\end{array}$ \\
\hline Ftape 3 de l'annés 3 & $\begin{array}{l}\text { Co-construire le diypositif } \\
\text { permettant de répondre aux } \\
\text { questions de recherche } \\
\text { Co-interpréter les doennées }\end{array}$ & $\begin{array}{l}\text { Co-constnire le dispositif } \\
\text { permettant de répondre aux } \\
\text { questions de recherche } \\
\text { Co-interpréter les doanées } \\
\text { Organiser les connaissances et les } \\
\text { diffucer }\end{array}$ \\
\hline
\end{tabular}

\subsection{LES OUTILS D'ANALYSE}

Les données collectées au cours des trois années de recherche ont été analysées dans une perspective doublement comparative. J'ai comparé les différentes versions du récit de chaque élève et tous les récits des élèves entre eux.

J'ai interprété les récits recueillis en utilisant la grille de Heinz (adaptée par Delory-Momberger, 2014, pp. 89-92), (Tableau 3), qui situe les interprétations au croisement des théories de l'expérience et de celles de l'action. J'y ai intégré le triptyque multiréférentiel « agent-acteur-auteur » proposé par Ardoino (1992). 
Tableau 3

Grille d'interprétation

\begin{tabular}{|l|l|}
\hline Formes du discours & Degré de mise en rénexivité du narrateur \\
\hline Schemas d'action et systèmes d'actants & $\begin{array}{l}\text { Manière dont le narrateur se positionne et } \\
\text { positionne les autres actants (actif, passif, agent, } \\
\text { acteur, auteur, ressource, obstacle...) }\end{array}$ \\
\hline Topoi, thèmes récurrents & $\begin{array}{l}\text { Organisation de l'action et du ressenti du } \\
\text { aarrateur, cles d'interprétation du vécu }\end{array}$ \\
\hline Gestion biographique des topoi & $\begin{array}{l}\text { Manière dont les narrateurs interprètent, } \\
\text { evaluent. ajustent leur action. }\end{array}$ \\
\hline
\end{tabular}

Les quatre axes d'analyse de la grille de Heinz, associées pour ce qui est des schémas d'action et des systèmes d'actants au tryptique multiréférentiel, m'ont permis d'avoir accès à une vision globale de la manière dont les élèves évaluaient et ajustaient leurs actions à la forme scolaire et dont ils configuraient en conséquence leurs figures d'élève pour produire des connaissances sur leurs modalités de subjectivation du parcours scolaire et d'orientation.

Des entretiens complémentaires menés avec les participants, à distance d'un à deux ans de la mise en œuvre du dispositif et portant sur leur participation à la recherche, ont permis d'en mesurer les effets à plus long terme et d'interroger la nature de la relation établie avec les élèves en vue de produire des connaissances et de transformer une situation insatisfaisante.

\section{EFFETS DU DISPOSITIF ET DISCUSSION}

\subsection{POUR LES ÉLÈVES}

La première remarque concerne les effets de la recherche sur les deux classes dans lesquelles j'avais le statut d'enseignante.

J'ai pu y observer un net changement du climat. Les élèves avaient tendance en début d'année à se regrouper par un jeu d'affinités variables (quartier de résidence, pays d'origine, genre, catégories socio-professionnelles des parents, loisirs pratiqués) et certains restaient isolés. Globalement, la cohabitation entre eux se faisait dans des tensions importantes, les élèves ou les microgroupes se plaçant en concurrence les uns par rapport aux autres.

Le travail de narration socialisée leur a permis de mieux se connaître et de prendre conscience qu'ils partageaient certaines souffrances sans le savoir, comme en témoignent ces extraits d'entretien, enregistrés en fin de séance, au moment du bilan du travail :

Vuong: Ben on a des... des histoires différentes, mais c'est un peu pareil.

Raphaël: Et puis, y a des trucs on savait pas, maintenant on sait, pour mieux se comprendre, quoi.

Sébastien: Ouais. Et j'ai vu que y avait pas que moi aussi qui était comme moi. $\mathrm{Y}$ avait d'autres élèves aussi.

Alicia: Ben, en fait on est tous un peu pareils, on s'en rendait pas compte.

Linda: Je trouve que ça permet de voir que les autres aussi ont des problèmes. 
Mais c'est aussi la découverte et la compréhension des singularités, des différences, leur connaissance et leur reconnaissance qui a permis aux élèves de s'accepter et de commencer à coopérer les uns avec les autres.

Ils ont progressivement changé d'attitude et il m'a été de plus en plus facile de proposer des activités autogérées. Les élèves parvenaient de mieux en mieux à communiquer, dans un plus grand respect des uns et des autres. Ils se sont constitués en communauté de travail et d'objectifs, se montrant solidaires les uns des autres pour que chacun puisse progresser.

Ils ont aussi, en plus du fait qu'ils se sont activement engagés dans une réflexion sur leur orientation, fait preuve de solidarité au moment des recherches de stages. Cela n'a été possible que parce qu'ils s'étaient au préalable tous découverts et reconnus comme membres d'un groupe ayant des intérêts communs. Bien entendu, ils ne se sont pas tous transformés en "élèves modèles », et ont continué à vivre d'importants conflits identitaires. Mais globalement, la posture réflexive adoptée a permis l'instauration d'une ambiance propice au travail et à l'élaboration de projets individuels et collectifs.

La deuxième remarque concerne cette fois les trois premiers groupes qui ont participé à la recherche. Les élèves se sont globalement engagés plus tôt et de façon plus active dans une réflexion sur leur avenir. Ils ont gagné en autonomie, se rendant plus nombreux que les cohortes précédentes aux portes ouvertes des lycées professionnels, cherchant et trouvant seuls des stages en entreprise.

Les bilans scolaires réalisés avec les équipes éducatives ont mis en évidence qu'ils avaient pour la plupart adopté une attitude plus positive et confiante à l'égard de leur scolarité, et qu'ils étaient entrés dans une posture plus stratège, cherchant à faire entrer en adéquation leurs résultats et leurs projets.

Par ailleurs, et cela concerne cette fois tous les groupes qui se sont impliqués dans la recherche, les élèves ont pu prendre conscience de certains déterminismes familiaux ou sociaux dans lesquels s'inscrivaient leurs parcours et les mettre à distance.

En témoignent par exemple ces propos de Mélina, recueillis plus d'un an après la mise en œuvre du dispositif dans la classe « expérimentale » :

Ben, en fait, ce que ça m'a apporté aussi, c'est qu'avant, je croyais que c'était moi qui était nulle. Mais en écoutant tout le monde et puis en réfléchissant, tout ça, c'que j'ai compris c'est qu'en fait, c'est pas que moi. Même dans la classe y avait aussi Adriana qui venait d'Angola et comme moi, c'était dur pac'qu'elle parlait pas français en fait. Et même d'autres, Keti et Rima qui étaient tchétchènes, et même d'autres qui z'avaient été en foyer, ou je sais pas moi, qu'on pas eu de chances aussi... En fait quand j'ai compris que c'était pas moi, j'me suis dit que je pouvais, même si c'était pas facile, que j'étais pas plus bête et même si c'est difficile et quand même ce sera long, ben je vais y arriver. Maintenant j'le sais. 
Ou encore ceux de Camille, recueillis lors de l'entretien co-analytique, mis en œuvre la $3^{\text {¿̀me }}$ année qui mettent en relation les difficultés rencontrées avec l'établissement de scolarisation et son quartier d'implantation :

Ça a toujours une réputation... un peu de... y a des gens ils l'appellent le collège poubelle, c'est récupérer en fait, détruire les... les déchets des .... Ou alors l'inverse en fait, quand on se fait virer [d'un autre collège], ben on va là. En fait, c'est bizarre. En fait entre les deux collèges eux, y z'ont, y z'ont plus de chance, on dirait. En fait, je sais pas, c'est bizarre.

La participation à la recherche a manifestement amené les élèves à développer une distance critique par rapport à leur expérience et à développer un plus grand pouvoir d'agir sur leur parcours. Ils ont pu affirmer leur subjectivité, se positionnant davantage en auteurs dès lors qu'ils conscientisaient les influences sociales qui s'exerçaient sur eux, pour choisir d'écrire leur histoire en continuité ou en rupture avec celles-ci.

\subsection{POUR L'ENSEIGNANTE ET CHERCHEURE}

J'ai commencé ma recherche en tant qu'enseignante qui voulait à la fois comprendre un phénomène et transformer une situation. Un des premiers effets de mon travail est la construction d'une identité de chercheure, dissociée de mon identité d'enseignante, mais s'y adossant en quelque sorte. Elle s'inscrivait dans une continuité avec mon parcours de vie, orienté par des valeurs et un projet qui m'a mené successivement à enseigner, puis à me spécialiser dans la prise en compte de la difficulté scolaire, et enfin, à reprendre des études et entrer en recherche. Il s'agissait pour moi, tout au long de ce parcours, de contribuer à une éducation émancipatrice et égalitaire.

Les connaissances construites m'ont permis de mieux comprendre les enjeux de l'orientation pour les élèves et de la construction de figures d'élèves. J'ai ensuite pu accompagner de façon plus efficiente les élèves dans leur orientation mais aussi dans les apprentissages narratifs permettant d'interpréter son expérience et de se projeter dans l'avenir. Cela m'a menée, par exemple, à introduire de nouvelles activités pour permettre aux élèves de mutualiser les outils narratifs et à modifier les modalités d'évaluation en matière d'expression de soi pour mieux valoriser les élèves.

Globalement, j'ai pu reconstruire un sentiment de pouvoir agir dans l'intérêt de mes élèves et dans le sens de mes idéaux.

\subsection{CONDITIONS DU DÉVELOPPEMENT D'UNE POSTURE D'AUTEUR}

Il me semble important de dégager quels éléments du dispositif mis en œuvre dans le cadre de la RBE (visant, je le rappelle, l'émergence d'un sujet réflexif, lucide et responsable de ses choix) ont permis de développer une posture d'auteur. 
Le premier correspond à la manière d'envisager les participants à ma recherche. Ils ont d'emblée été envisagés comme des sujets, au sens éthique du terme, c'est-à-dire comme détenteurs d'une intelligence de la situation qu'ils vivaient et comme sujets du discours qu'ils tenaient. Cela impliquait que leur parole soit entendue et respectée, inconditionnellement. Ils ont également été considérés comme des sujets politiques en construction, responsables apprenant de leur orientation et par conséquent invité à faire des choix lucides pour interagir avec leur environnement.

La rencontre de la personne-chercheure-enseignante avec les personnes-participants à la recherche-élèves a englobé à la fois la situation de recherche et la situation d'enseignement pour dépasser les asymétries de relation présentes dans ces dernières, à partir du moment où les savoirs coconstruits n'ont pas été soumis à une hiérarchisation. La construction du savoir théorique a accompagné la production de connaissances empiriques sans la surplomber.

En effet, les méthodes mises en œuvre, destinées à constituer l'espace de recherche en un espace d' « auteurisation », ont permis de ne pas enfermer la rencontre dans un dispositif protocolaire préalablement conçu. Cela s'est traduit concrètement par la possibilité pour les élèves de se retirer de la recherche à tout moment, en demandant que leurs productions ne soient pas utilisées, en choisissant de modifier les données, ou encore en faisant des propositions en matière de recueil ou d'interprétation des données. Dans ces conditions, la recherche pouvait altérer positivement l'ensemble des acteurs.

Rhéaume (2019) met en évidence que le développement du pouvoir d'agir se déploie dans quatre dimensions : l'estime de soi, la reconnaissance des compétences, un cadre relationnel marqué par des rapports égalitaires ou visant l'égalité, le développement d'une conscience sociale critique (Rhéaume, 2019, p. 127). Les résultats du dispositif (Dizerbo, 2019) mettent en évidence que la présence de ces dimensions dans la recherche ont permis de développer le pouvoir d'agir de tous les acteurs et par conséquent de transformer des situations.

\section{CONCLUSION}

Dans la recherche relatée, les élèves étaient invités à interpréter ou réinterpréter leur parcours pour en envisager la suite en position d'auteurs. Les résultats ont démontré que la performativité du récit a contribué à les transformer, modifiant leur posture et leur relation au savoir et les faisant entrer dans un rapport plus lucide et stratège à leur scolarité. Mais cela n'a été possible que parce qu'une attention particulière était accordée à la construction d'une véritable rencontre relationnelle entre le chercheur et les acteurs-sujets de la recherche.

Dans les situations d'investigation s'inscrivant dans la RBE, qui visent la transformation d'une situation et le développement du pouvoir d'agir des participants, la finalité et la qualité de la relation établie apparaît en effet comme fondamentale. 
Il s'agit à tout moment de s'assurer que le dispositif garantit les possibilités d'une émancipation du participant et que sa démarche favorise le développement de son pouvoir d'agir. Cela n'est possible qu'en prêtant attention à la qualité de la relation établie.

Parce que le chercheur met en œuvre un dispositif dont il ne maîtrise pas l'issue, il se doit d'effectuer un travail important sur ses implications en conscientisant ses aprioris, pour suspendre dans la rencontre tout jugement et entrer dans une écoute empathique qui invite à " se décentrer de soi, et par conséquent, à accepter de changer, d'être transformé » (Janner Raimondi, 2019, pp. 74-75).

Au prix de ce travail, il pourra finalement accéder à une compréhension herméneutique de l'expérience des participants, dans un processus hétérobiographique, c'est-à-dire se situant au carrefour d'une lecture-écriture de son histoire et de celle des participants, dans une relation d'humain à humain avant tout. Elle caractérise le paradigme de la RBE, situé dans le prolongement de l'épistémologie fondée par Dilthey.

La dimension éthique et politique de la RBE, qui apparait dans la volonté et la responsabilité qu'elle se donne de rendre visible et de transformer l'expérience des sujets, en constituant la situation de recherche comme une adresse de personnes à personnes, implique en effet l'exercice constant d'un sens critique. Delory-Momberger (2014) rappelle à ce propos que :

Sur le plan de l'éducation, ce rapport à l'autre que soi a été en particulier problématisé par Fichte qui développe l'idée que l'éducation n'est formation de l'homme que parce qu'elle est, non pas une action sur un objet, mais une action réciproque entre des êtres raisonnables et libres qui se reconnaissent comme des personnes. Chacun ne devient un sujet libre que parce qu'un autre sujet l'appelle à agir librement en le traitant déjà comme un sujet libre et parce qu'à son tour il traite cet autre sujet comme un sujet libre. (p. 65)

Ce principe s'applique à l'éducation mais aussi plus largement à la RBE, parce que tout en cherchant à comprendre l'expérience des sujets, elle vise des transformations individuelles qui ouvrent des potentialités de changements sociaux dont la perspective se trouve la motivation première des recherches mises en œuvre dans son cadre.

\section{RÉFÉRENCES}

Ardoino, J. (1992). L'implication. Voies Livres.

Ardoino, J. (2000). Les avatars de l'éducation. Presses Universitaires de France.

Bruner, J. (2010). Pourquoi racontons-nous des histoires? Retz.

Delory-Momberger, C. (2009). La condition biographique. Essais sur le récit de soi dans la modernité avancée. Téraèdre.

Delory-Momberger, C. (2014). De la recherche biographique en éducation. Fondements, méthodes, pratiques. Téraèdre. 
Dizerbo, A. (2019). Paroles d'élèves et bonheur à l'école. Tréma [En ligne], 52. https://doi.org/10.4000/trema.5504

Dominicé, P. (1999). La compétence d'apprendre à l'âge adulte: Lectures biographiques des acquis de la scolarité. Cahiers de la Section des Sciences de l'Éducation, 87, 1-21.

Janner Raimondi, M. (2019). Empathie. In C. Delory-Momberger (Coord.), Vocabulaire des histoires de vie et de la recherche biographique (pp. 72-75). Érès.

Larrosa, J. (1998). Apprendre et être. ESF.

Boutinet, J. P. (2007) (Dir.), Penser l'accompagnement adulte. Presses Universitaires de France.

M'Biatong, J. (2019). La recherche biographique en éducation pour l'éthique. In A. Dizerbo \& J. M'Biatong (Coord.), Pour une éthique de l'accompagnement biographique (pp. 129-152). Téraèdre.

Rhéaume, J. (2019). Pouvoir d'agir. Empowerment. In C. Delory-Momberger (Coord.), Vocabulaire des histoires de vie et de la recherche biographique (pp. 127-129). Érès.

Ricœur, P. (1990).Soi-même comme un autre. Seuil.

Tap, P. (1980). Identité individuelle et personnalisation. Privat. 
i Centre de Recherche Interuniversitaire Expérience Ressources Culturelles Éducation, Université Sorbonne Paris Nord, France. EXPERICE-EA3971

https: //orcid.org/0000-0001-5815-1950

Toda a correspondência relativa a este artigo deve ser enviada para:

Anne Dizerbo

1 allée Marie Le Vaillant

22000 Saint-Brieuc

dizerboanne@sujetdanslacite.org

Recebido em 23 de março de 2020

Aceite para publicação em 7 de julho de 2020 


\title{
Relation with subjects in biographical research in education
}

\begin{abstract}
This contribution intends to question the relationship of the researcher with the actors-subjects of research, with regard to the specificities of biographical research in education and the effects of the devices it implements. It is based on research carried out with several groups of middle school students and based on collective and individual biographical interviews and on written accounts of their school career. This research had two objectives: to understand the modalities of subjectivation of their educational path and orientation; to transform a situation in which the students had difficulties taking ownership of their path and orientation and in cooperating. This involves presenting the implemented device as well as its epistemological and methodological framework then of analyzing the effects to understand the stakes of the relationship of the researcher with the actors-subjects in biographical research. It will be highlighted that the specificities of this field of research invite, on the one hand, to register the investigation in a symmetrical meeting space, authorization space, favourable to the development of the power to act of the actors, and, on the other hand, to develop an empathetic posture guaranteeing the respect for the actors and their empowerment.
\end{abstract}

Keywords: Biographical research in education; Posture; Subject; Empowerment. 


\section{A relação com os sujeitos na investigação biográfica em educação}

\section{RESUMO}

Esta contribuição visa questionar a relação do investigador com os atores-sujeitos da investigação, a partir da especificidade da investigação biográfica em educação e dos efeitos dos dispositivos que implementa. A análise apoia-se numa investigação realizada com vários grupos de jovens do ensino básico, através de entrevistas biográficas coletivas e individuais, e de narrativas escritas, centradas no seu percurso escolar. A investigação teve duas finalidades: compreender as modalidades de subjetivação do seu percurso escolar e de orientação; transformar uma situação em que os alunos apresentam dificuldades, através da apropriação do seu percurso de orientação e da cooperação. Trata-se de apresentar o dispositivo usado, o seu quadro epistemológico e metodológico, para analisar os seus efeitos e compreender os desafios que se colocam à relação entre o investigador e os atores-sujeitos em investigação biográfica. Procuramos evidenciar que as especificidades deste campo de investigação convidam, por um lado, a inscrever a pesquisa no espaço de um reencontro simétrico, espaço de autorização, propício ao desenvolvimento do poder de agir dos atores e, por outro lado, a desenvolver uma postura empática garante do respeito pelos atores e pela sua autonomização.

Palavras-chave: Investigação biográfica em educação; Postura; Sujeito; Poder de agir. 